\title{
STRESSING STATE ANALYSIS OF LARGE CURVATURE CONTINUOUS PRESTRESSED CONCRETE BOX-GIRDER BRIDGE MODEL
}

\author{
Jun $\mathrm{SHI}^{1}$, Jiyang SHEN ${ }^{2,3}$, Guangchun $\mathrm{ZHOU}^{2,3^{*}}$, Fengjiang QIN ${ }^{4}$, Pengcheng $\mathrm{LI}^{2,3}$ \\ ${ }^{1}$ School of Transportation Science and Engineering, Harbin Institute of Technology, Harbin 150090, China \\ ${ }^{2}$ Key Lab of Structures Dynamic Behavior and Control of the Ministry of Education, \\ Harbin Institute of Technology, Harbin 150090, China \\ ${ }^{3}$ Key Lab of Smart Prevention and Mitigation of Civil Engineering Disasters of the Ministry of Industry \\ and Information Technology, Harbin Institute of Technology, Harbin 150090, China \\ ${ }^{4}$ Key Laboratory of New Technology for Construction of Cities in Mountain Area, School of Civil Engineering, \\ Chongqing University, Chongqing 400030, China
}

Received 19 December 2018; accepted 05 March 2019

\begin{abstract}
This paper experimentally analyzes the working behavior characteristics of a large-curvature continuous prestressed concrete box-girder (CPCBG) bridge model based on structural stressing state theory. First, the measured strain data is modeled as generalized strain energy density (GSED) to characterize the stressing state of the bridge model. Then, the Mann-Kendall (M-K) criterion is adopted to detect the stressing state leaps of the bridge model according to the natural law from quantitative change to qualitative change of a system, which derives the new definition of structural failure load. Correspondingly, the stressing state modes for the bridge model's sections and internal forces are proposed to verify their changing characteristics and the coordinate working behavior around the characteristic loads. The analytical results reveal the working behavior characteristics of the bridge mode unseen in traditional structural analysis, which provides a new angle of view to conduct structural analysis and a reference to the improvement of design codes.
\end{abstract}

Keywords: stressing state, mutation, failure load, stressing state mode, prestressed concrete box-girder bridge.

\section{Introduction}

In recent years, the continuous curved girder bridge has been increasingly favored by engineers due to its economic, aesthetic and increasing need of urban overpass construction. Also, steel structures and steel-concrete composite structures were usually adopted in the construction of curved girders (Kim \& Yoo, 2006; Yuan, Dai, \& Sun, 2013; Rogers \& Seo, 2016). Undoubtedly, the latter was more complicated owing to the addition of concrete (Przemysław, Wojciech, \& Radomski, 2017), so that considerable researches were contributed to reinforced concrete curved girder bridges. But so far analytical methods have not met the requirement of engineering applications in accuracy. Consequently, some unexpected accidents, such as the collapse accident on the Yuegan Highway in China, took place due to the absence of necessary researches for design reference (Shi, Cao, Ma, \& Ruan, 2018). Actually, theoretical and experimental analyses of curved girders could be retraced as early as 1930s (Timoshen- ko \& Gere, 1961). Vlasov (1961) established the basic differential equation of the curved girder with rigid section and proposed the generalized coordinate method for the constraint torsion theory of thin-walled bar member and then Dabrowski (1968) studied the bending and torsional behavior of thin-walled beams with asymmetric sections. With the development of computer technology, the finite strip method of curved plate structures and curved box girders was put forward by Meyer and Scordelis (1970); afterwards, M. S. Cheung and Y. K. Cheung (1984) extended it to the analysis of box girders with variable section.

The progress of construction techniques and analytical methods promoted the prestressed concrete girder bridges with the advantages of high strength and small section area. It was verified that prestress greatly improved the rationality of stress distribution for the curve concrete bridge and enhanced its spanning capacity (Lin \& Burns, 1983). But, because of the existence of planar curvature, the prestress could make the stress distribution in the structure

${ }^{*}$ Corresponding author. E-mail: gzhou@hit.edu.cn

\#Corresponding author. E-mail: qinfengjiang@cqu.edu.cn 
more complicated and would produce some adverse effects (Walter, 1985). Later, the relative researches started to involve the complication of prestressed curved bridges. In the recent decade, both experimental and simulative means became conventional in the research on prestressed curved bridges. Khaloo and Kafimosavi (2007) studied flexural behavior of horizontally curved prestressed box bridges using the refined 3-D finite element (FE) model and indicated that the redistribution of prestressed tendons across the section width could reduce critical stresses substantially. Huang, Liu, Zhang, and Tian (2009) experimentally and numerically analysed the characteristics of reacting forces inside and outside a three span prestressed concrete curved girder bridge. Yang, Zhao, and Liu (2011) simulated the stretching of prestressed tendons of the bridge model and put forward the optimal stretching sequence in the basis of comparison. Qiao, Jin, Tian, and Li (2012) investigated the coupled bending-torsion and shear lag effects through the deduced differential equation of curved prestressed box girders with different boundary conditions, which coincided with the experimental and simulative results well. Besides, a few scholars carried out the researches on the stressing state of large curvature CPCBG bridges in their fully loading process (Jiang, 2008; Cai, 2013). Recently, some researchers focused on construction monitoring, FE modeling approaches for seismic evaluation, and so on ( $\mathrm{Bu} \&$ Zheng, 2017; Seo \& Rogers, 2017; Seo, Rogers, \& Hu, 2018). Also, Khan, Lobo, and Linzell (2018) tested and simulated the live load performance of a curved prestressed concrete transit rail bridge, which demonstrated the effects of speed and centrifugal forces on dynamic amplification and live load distribution. Although these research results greatly promoted the application of large curvature CPCBG bridges, two problems in the working behavior of prestress curved box girder bridges have been puzzling researchers and limiting their development to an extent:

1. The existing analytical theories and methods are difficult to achieve an accurate prediction of structural load-bearing capacity, in view of the complex stressing state of curved bridge structures with prestress. As a result, the semi-empirical and semi-theoretical methods for determining the ultimate bearing capacity of the bridge structures are in fact to avoid and minimize the negative effects derived by the inaccurate prediction. This could lead to considerable material cost and even irrationality in structural design due to the negative effects.

2. As a curved bridge structure involves various parameters and complicated failure mechanism, many an experiment is required to investigate its working behavior. But, the high experimental cost brings about insufficient experimental data for the existing analytical methods and calculating formulas. Importantly, the existing theories and methods could not be fully applied or might lack the abilities to deeply reveal the unseen knowledge in the even limited experimental data.
Hence, it is significant and vital to develop the innovative theory and method to address the issues. This paper experimentally investigates the whole working process of a large curvature CPCBG bridge model, based on the new concept of structural stressing state and the corresponding analytical method, aiming at revealing some unseen stressing state characteristics in the experimental data.

\section{Modeling of structural stressing state and $\mathrm{M}-\mathrm{K}$ criterion}

\subsection{Structural stressing state concept}

Structural stressing state is the inner and outer modes presented by the responses of components/units or their combinations in a structure under a certain loading case, as defined by Zhou, Rafiq, Bugmann, and Easterbrook (2006) and Zhou, Pan, Xu, and Rafiq (2010). Structural stressing state is generally for the whole structure and stressing state submodes for individual structural components, local parts and internal forces. Structural stressing state mode can be expressed by the matrix or the vector consisting of structural response data such as strain, stress, strain energy, displacement and internal force.

Structural stressing state will change/evolve with the load increase and embodies different characteristics at some special load levels, according to the natural law from quantitative change to qualitative change of a system (Zhang, Zhou, Xiong, \& Rafiq, 2010). Actually, structural stressing state will present the qualitative mutation characteristic (shape change or magnitude mutation of stressing state mode) from the quantitative change (stable and small magnitude change of stressing state mode) once the load reaches to a certain level. Actually, various types of structures with different failure forms certainly have this essential and common mutation characteristic of structural stressing state, which can be distinguished by the proposed criteria. Meanwhile, the stressing state submodes can present the feature of bifurcation besides their individual mutation characteristics. The mutation characteristics of structural stressing state will reveal the starting point of structural failure and the structural failure mechanism, leading to the update of the existing analytical theories and analytical methods as well as the more rational design codes.

\subsection{Numerical description of structural stressing state}

As introduced above, the stressing state of a structure can be expressed by the response data of key points from the experimental measurement or numerical simulation. In order to construct the numerical mode (vector or matrix) of structural stressing state and the parameter characterizing it, generalize strain energy density (GSED) as a scalar is adopted to describe the stressing state of a point. Since the response data has the linear-elastic, elastic-plastic and even plastic differences, the formula for calculating structural elastic response is generalized to model the stressing state of a point, that is, Eqn (1) is utilized in form to yield 
the GSED value of the ith point:

$$
E_{i}=\int \sigma_{1} d \varepsilon_{1}+\sigma_{2} d \varepsilon_{2}+\sigma_{3} d \varepsilon_{3},
$$

where $\sigma_{1}, \sigma_{2}, \sigma_{3}$ and $\varepsilon_{1}, \varepsilon_{2}, \varepsilon_{3}$ are three principal stresses and strains, respectively; $E_{i}$ is the GSED value of the $i$ th point. Also, the measured strains can be generalized to describe the stressing state of a point:

$$
E_{i}=\frac{1}{2} \sum_{j=1}^{N} E^{\prime} \varepsilon_{j}^{2},
$$

where $E_{i}$ is the GSED value of the $i$ th point; $\varepsilon_{j}$ is the $j$ th principal strain; and $E^{\prime}$ is nominal elastic modulus. Thus, structural stressing state mode can be formed by the GSED values of key points in the structure, as a vector or a matrix. Accordingly, the GSED sum $E$ at each load level, as the parameter characterizing structural stressing state, can be calculated by

$$
E=\sum_{i} E_{i}
$$

where $E_{i}$ is the GSED value of the $i$ th key point at load level $F$. Furthermore, the $E-F$ curve can be plotted to investigate structural stressing state characteristics vividly.

\subsection{Mann-Kendall criterion}

Mann-Kendall (M-K) criterion is a widely used trend analysis tool normally without necessity for samples to comply with certain distributions or interference of a few outliners (Mann, 1945; Hirsch, Slack, \& Smith, 1982; Kendall, 1990). Applying the M-K criteron, the mutation of structural stressing state can be distinguished through the $E-F$ curve (Huang, Yu, \& Liu, 2014). Assume that the sequence of $\{E(i)\}$ (the load step $i=1,2, \ldots, n)$ is statistically independent. Then, a statistical quantity $d_{k}$ at the $k$ th load step can be defined as

$d_{k}=\sum_{i}^{k} m_{i}(2 \leq k \leq n), m_{i}= \begin{cases}+1 & E^{\prime}(i)>E^{\prime}(j)(1 \leq j \leq i), \\ 0 & \text { otherwise }\end{cases}$

where $m_{i}$ is the cumulative number of the samples; " +1 " means adding one more to the existing value if the inequality on the right side is satisfied for the $j$ th comparison. Calculate the mean value $E\left(d_{k}\right)$ and the variance $\operatorname{Var}\left(d_{k}\right)$ of $d_{k}$ :

$$
\begin{aligned}
& E\left(d_{k}\right)=k(k-1) / 4(2 \leq k \leq n) ; \\
& \operatorname{Var}\left(d_{k}\right)=k(k-1)(2 k+5) / 72(2 \leq k \leq n) .
\end{aligned}
$$

Then, a new statistic quantity $U F_{k}$ is defined by

$$
U F_{k}= \begin{cases}0 & k=1 \\ d_{k}-E\left(d_{k}\right) / \sqrt{\operatorname{Var}\left(d_{k}\right)} & 2 \leq k \leq n\end{cases}
$$

and the $U F_{k}-F$ curve can be plotted.

For the inverse sequence of $\{E(i)\}$, the same steps from Eqn (4) to Eqn (7) are proceeded to derive the $U B_{k}-F$ curve. Thereby, the characteristic point of the $E^{\prime}-F^{\prime}$ curve, i.e., the mutation of structural stressing state, is determined by the intersection of the $U F_{k}-F$ and $U B_{k}-F$ curves.

\section{Experimental bridge model}

\subsection{Configuration of the bridge model}

A large-curvature CPCBG bridge model was designed and tested by Dai (2007) in Chang'an University in China. The bridge model is scaled as about 1:5 based on the similarity theory and satisfies the requirements of geometrical similarity, boundary conditions and physical conditions, which can certainly reflect the main mechanical characteristics of the actual bridge (Zhang, 2002; Shi \& Xiang, 2012). The bridge model has a single-box and single-chamber cross section with a total span of $12 \mathrm{~m}$ along the bridge centerline and a radius of curvature of $10 \mathrm{~m}$. The centerline of the bridge model corresponds to a central angle of $68.75^{\circ}$ $(1.2 \mathrm{rad})$ and the cross sections are shown in Figure 1. An $800 \mathrm{~mm}$ long variable cross-section component is set between the beam section and the typical cross-section. The yield stress, elastic modulus and fracture strength of steel bars are shown in Table 1. The tensile strength and elastic modulus of prestressed tendons are $1860 \mathrm{MPa}$ and $195 \mathrm{GPa}$. The compressive strength and elastic modulus of concrete are $52.12 \mathrm{MPa}$ and $37.79 \mathrm{GPa}$. Taking the centerline of the bridge mode as the symmetrical axis, the side far from the center of the circle is the "outer side" and the other one is the "inner side". Figure 1 shows the experimental model and the testing apparatus with six parts. The arch model and abutment are connected by the transition section. Abutments are fixed on the ground using anchor blocks. Tie-rods between abutments are used to balance the horizontal thrust from arch feet. The out-of-plane deformation of the arch is limited by lateral confinements. To simulate the process of loading case 3 , five loading devices are set at equal intervals along the arch span where the jacks exert controllable loading process.

Table 1 . The material property of steel bars

\begin{tabular}{|c|c|c|c|}
\hline $\begin{array}{c}\text { Steel bar } \\
\text { diameter } \\
(\mathrm{mm})\end{array}$ & $\begin{array}{c}\text { Yield stress } \\
(\mathrm{MPa})\end{array}$ & $\begin{array}{c}\text { Elastic modulus } \\
(\mathrm{GPa})\end{array}$ & $\begin{array}{c}\text { Fracture strength } \\
(\mathrm{MPa})\end{array}$ \\
\hline 5 & 413.05 & 216 & 575.31 \\
\hline 10 & 512.05 & 214 & 790.04 \\
\hline
\end{tabular}

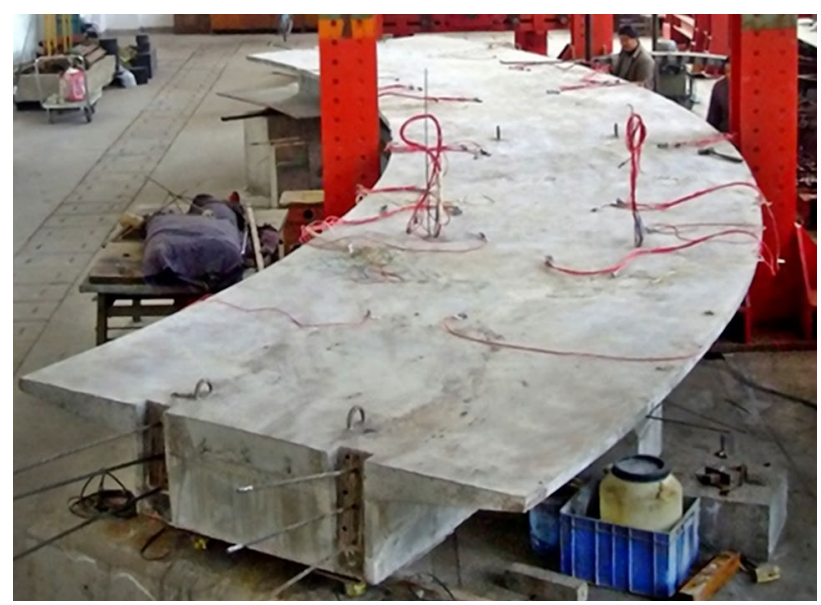

Figure 1. The large-curvature PCCBG bridge mode 


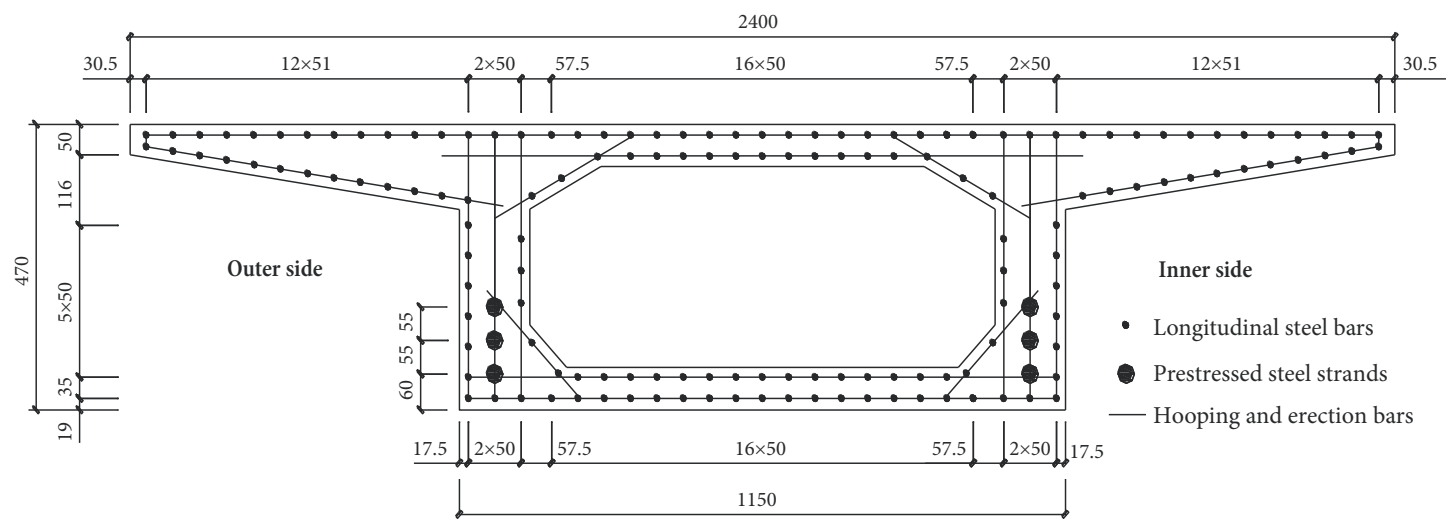

Figure 2. The layout of steel bars in the box girder model (Unit: $\mathrm{mm}$ )

\subsection{Layout of steel bars}

Figure 2 shows the layout of steel bars in the box girder. Longitudinal bars, stirrup, anti-collapse steel bars, etc. are set in the model box girder and the minimum spacing of steel bars is $5 \mathrm{~cm}$.

\subsection{Loading scheme and measuring arrangement}

Model girder is mounted on the $0 \# \sim 2 \#$ pier through rubber bearings and sensors. The vertical loads are exerted at two midspan locations, $2.3 \mathrm{~m}$ away from the center of the middle bearing, as shown in Figure 3. In the full loading process, the load is firstly applied with a constant increment of $100 \mathrm{kN}$ until $1000 \mathrm{kN}$ and then $50 \mathrm{kN}$ until the ultimate load, since the bridge model presents a relatively stable response process. Data collection starts after holding the load for 15 minutes each loading step and each reading/recording time is about $10-15$ minutes. An electron microscope with an accuracy of $0.01 \mathrm{~mm}$ is used to measure the crack width shown in Figure 3. Displacement and strain are measured at mid-span, mid-fulcrum and $\mathrm{L} / 3$ cross sections along the longitudinal direction of the bridge mode, as shown in Figure 3. The measuring plan on cross section is shown in Figure 4. Strain gauges are set on the concrete surface and embedded in concrete. Also, the strain gauges are put on the steel bars corresponding to the measuring points of concrete. The displacement meters are arranged at the bottom of the bridge model.

The first cracks occur on the soleplate at $550 \mathrm{kN}$ for the cross-loading section and on the roof surface at $750 \mathrm{kN}$ for the mid-fulcrum section; then the cracks at all sections continuously propagate and increase. When load is increased to $1000 \mathrm{kN}$, three cracks run throughout the entire soleplate at the cross-loading section in the first span, with a maximum width of $0.1 \mathrm{~mm}$. Four soleplate cracks develop to the web and the maximum width of the web crack is $0.08 \mathrm{~mm}$. The transverse crack of the mid-fulcrum develops throughout the entire roof and the maximum crack width is $0.14 \mathrm{~mm}$. When load is up to $1750 \mathrm{kN}$, the new cracks on the soleplate near the loading cross section continue to appear with the maximum crack width of $0.6 \mathrm{~mm}$, and the original cracks continue to propagate upward along the web. At the mid-fulcrum section, the web cracks densely distribute and the main crack width in the negative moment zone reaches to $1.5 \mathrm{~mm}$, which can be considered as the ultimate bearing state of the bridge model.

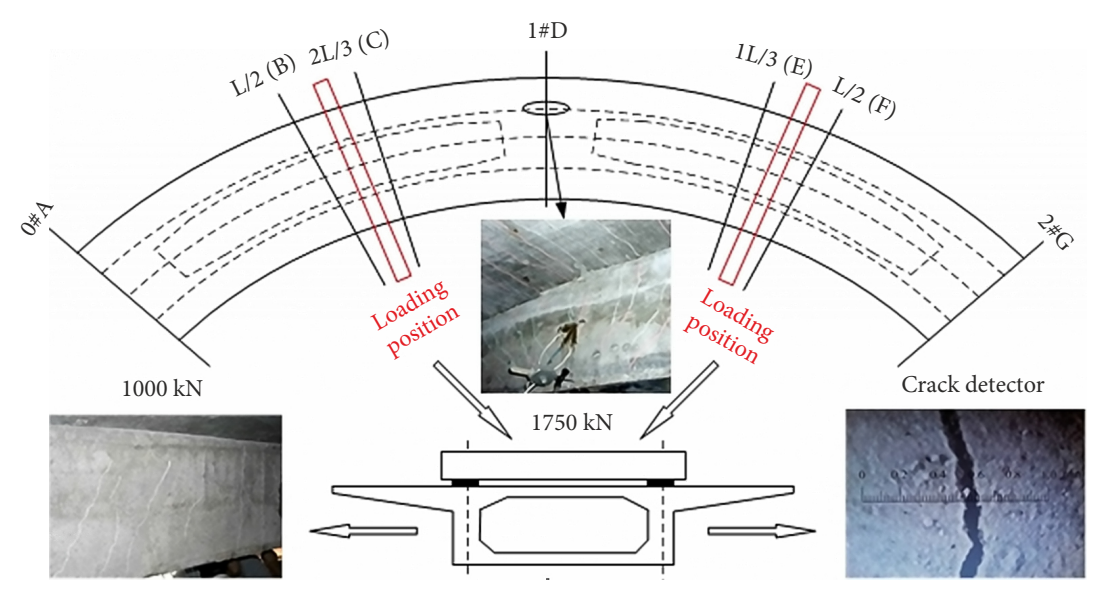

Figure 3. The loading positions and the crack profile of key locations 


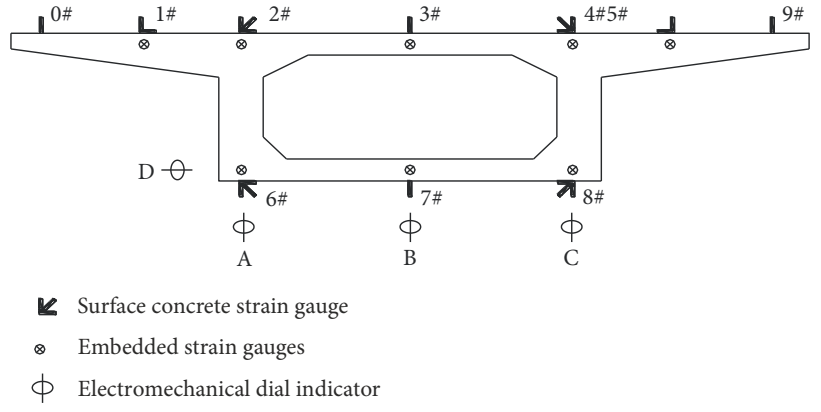

Figure 4. Strain gauges on concrete surface and embedded in concrete and steel bars as well as layout of displacement meters

\section{Characteristics of the bridge model's stressing state}

The experiment strain data is applied to model the characteristic pairs of structural stressing state, i.e., stressing state mode and characteristic parameter. Then, the M-K criterion and other judgments are adopted to detect the changing features of the characteristic pairs with load increase. Next, the essential leap feature of structure stressing state can be verified to be the starting point during structural failure process, leading to the update of the existing structural failure load. Finally, the deep structural analysis and the improvement of design codes could be conducted according to the results achieved by the structural stressing state analysis of the bridge model. The procedure of structural stressing state analysis for the bridge model is shown in Figure 5.

\subsection{GSED-based stressing state mode and characteristic parameter}

In order to reflect the structural stressing state features of the bridge model, the GSED values of the key points on the cross sections B and D at individual load levels $\left(F_{j}\right)$ are assembled to form the matrix expressing the stressing state mode, $\mathbf{s}_{j}=\left[\begin{array}{ccc}e_{\mathrm{B} 1} & \cdots & e_{\mathrm{BN}} \\ e_{\mathrm{D} 1} & \cdots & e_{\mathrm{DN}}\end{array}\right]_{j}$, in which $e$ is the GSED value of a point among $N$ measured points on cross section $\mathrm{B}$ or $D$. The parameter characterizing structural stressing state $\mathbf{S}_{j}$ is proposed as the sum of the GSED values:

$$
E_{j}=\sum_{i=1}^{N} e_{i j}
$$

where $e_{i j}$ is GSED value of the $j$ th key point to the $j$ th load level. So far, the modeling of structural working behavior based on the theory for analyzing structural stressing state has derived the stressing state mode $\mathbf{S}_{j}$ and its characteristic parameter $E_{j}$. The following investigation into the change of $\mathbf{S}_{j}$ and $E_{j}$ with load increase will reveal the leap feature of the bridge model's stressing state.

\subsection{Parameter characterizing structural stressing state}

The $E_{j}-F_{j}$ (written as $E-F$ below) curve can be plotted to reveal the changing feature of structural stressing state.

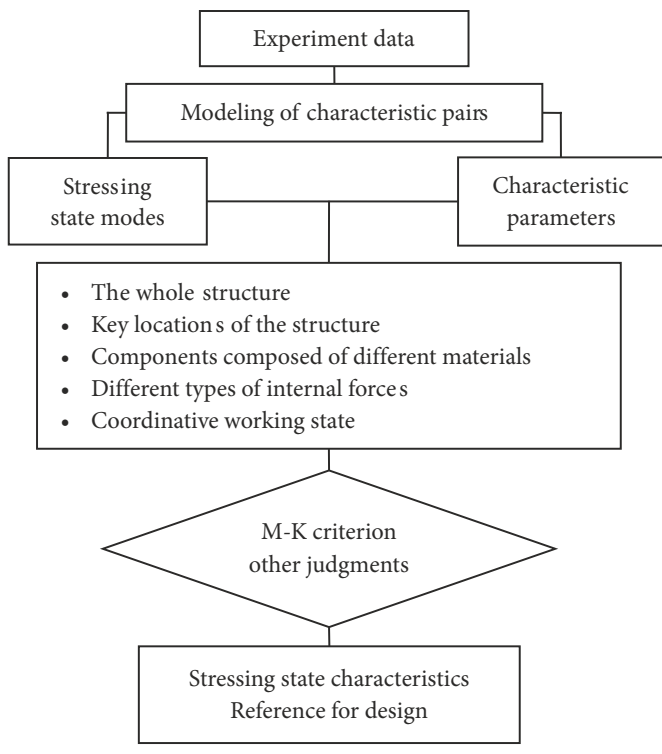

Figure 5. A flowchart of the stressing state analysis of bridge model

All measured strains are utilized to the calculation of Eqn (7). Then, two leap points of structural stressing state, $P=1050 \mathrm{kN}$ (from 0 to $U$ ) and $Q=1450 \mathrm{kN}$ (from $P$ to ultimate load $U$ ), are distinguished by the $\mathrm{M}-\mathrm{K}$ criterion, as shown in Figure 6. It is obvious that $E$ increases slowly before load $P$, indicating that the whole structure is in the elastic working state. After load $P, E$ increases within a narrow range probably due to the intensive development of concrete cracks and the reduction of sectional stiffness, speculating that the plastic deformation of the bridge model is large enough to affect the working behavior of the whole structure. From load $Q$ on, $E$ develops sharply and faster in a trend different from the previous one, implying that the bridge model goes into an unstable stressing state thoroughly different from the stable one, even though it can still bear more loads. Therefore, load $Q$ is defined as the failure load of the bridge model, which is the update of the existing failure load defined at structural ultimate bearing state. The updated failure load reveals the start point of the bridge model's failure process and has the attribution of certainty unlike structural ultimate load with indeterminate attribution. Since any load in the process of structural failure from the updated failure load to

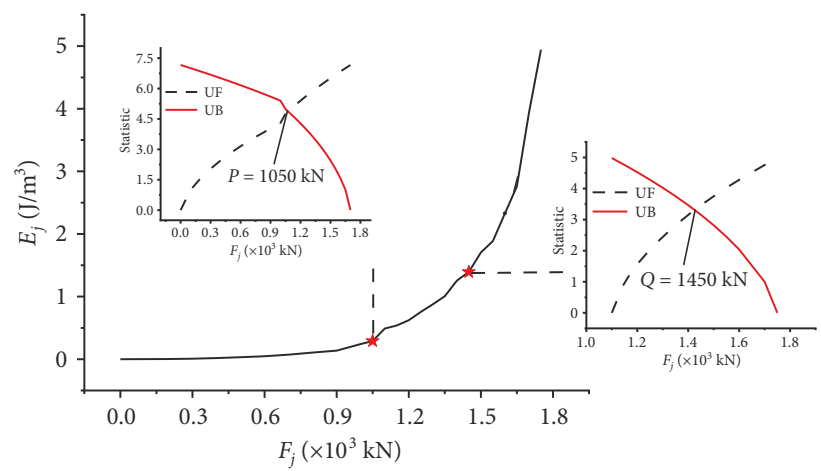

Figure 6. The $E_{j}-F_{j}$ and $\mathrm{M}-\mathrm{K}$ statistic curves 
the ultimate load cannot be allowed for the normal working (service) state of a structure, the updated failure load should be taken as the reference to the determination of structural design load. Besides, the updated failure load will lead to a more accurate and rational design load because of its attribution of certainty than that referring to the ultimate load with attribution of uncertainty.

Characteristic loads $P$ and $Q$ can divide the structural stressing state of the bridge model into three stages:

1. Before load $P$, the bridge model keeps a stable stressing state so that load $P$ is defined as the demarcation point between structural elastic and elastic-plastic stressing states;

2. After load $P$, the bridge model goes into the elasticplastic and still stable stressing state until load $Q$;

3. From load $Q$ on, the bridge model's stressing state suddenly and sharply changes until the ultimate load $U$.

The following investigation into structural stressing state mode will further verify the stressing state features embodied in the $E-F$ curve.

\section{Analysis of stressing state modes}

For the bridge model, cross section B or D suffers the larger response than the other cross sections. In other words, the stressing state mode for cross section B or D is not only the stressing state mode for the cross section itself but also the submode of the bridge model's stressing state. Hence, the stressing state mode for cross section B is investigated to see whether or not it also has the stressing state feature similar to that of the whole bridge model.

The stressing state mode of cross section B can be composed of the GSED values or strains at measured points shown in Figure 4, for instance, the GSED-based stressing state mode at different key point $\mathbf{S}_{\mathrm{cs}}=\left[e_{\mathrm{cs} 1}, e_{\mathrm{cs} 2}, \ldots, e_{\mathrm{cs} 8}\right]^{\mathrm{T}}$ for concrete at the cross section edge, $\mathrm{S}_{\mathrm{ci}}=\left[e_{\mathrm{ci} 1}, e_{\mathrm{ci} 2}, \ldots, e_{\mathrm{ci} 8}\right]^{\mathrm{T}}$ for concrete in the cross section and $\mathbf{S}_{\mathrm{ss}}=\left[e_{\mathrm{ss} 1}, e_{\mathrm{ss} 2}, \ldots, e_{\mathrm{ss} 8}\right]^{\mathrm{T}}$ for steel bars in the cross section. Thus, the $\mathbf{S}-F$ curves can be plotted to observe the changing feature of structural stressing state mode $\mathbf{S}$ with the increase of load $F$. The following investigation constructs different categories of structural stressing state modes or submodes to observe their changing features. The stressing state modes are various in form and depend on what structural behavior characteristic to investigate or what question to answer. In this study, structural leap features are concerned so that the stressing state modes are built by strains, GSEDs and displacements for key cross sections and different components (surface concrete, internal concrete and steel bars). Correspondingly, the characteristic parameters are derived to reflect the changing features of stressing state modes with load increase.

\subsection{Stressing state modes for different components}

Figure 7 shows the $\mathbf{S}_{\mathrm{GSED}}-F$ and $\mathbf{S}_{\text {strain }}-F$ curves of the stressing state modes $\mathbf{S}_{\mathrm{GSED}}$ and $\mathbf{S}_{\text {strain }}$ composed of
GSEDs and strains with respect to load level $F$. Three types of stressing state modes for the key cross section B are built according to the configuration of the bridge model, i.e., the stressing state modes for surface concrete, internal concrete and steel bars, respectively. For simplicity, the GESD-based stressing state modes for internal concrete and steel bars are not shown as their similarity to the strain-based ones. From Figure 7, the following features can be observed:

1. Figure 7(a) and (b) show that the stressing state modes have the leap features at characteristic loads $P$ and $Q$, consistent with that revealed from the characteristic parameter $E$ in Figure 6;
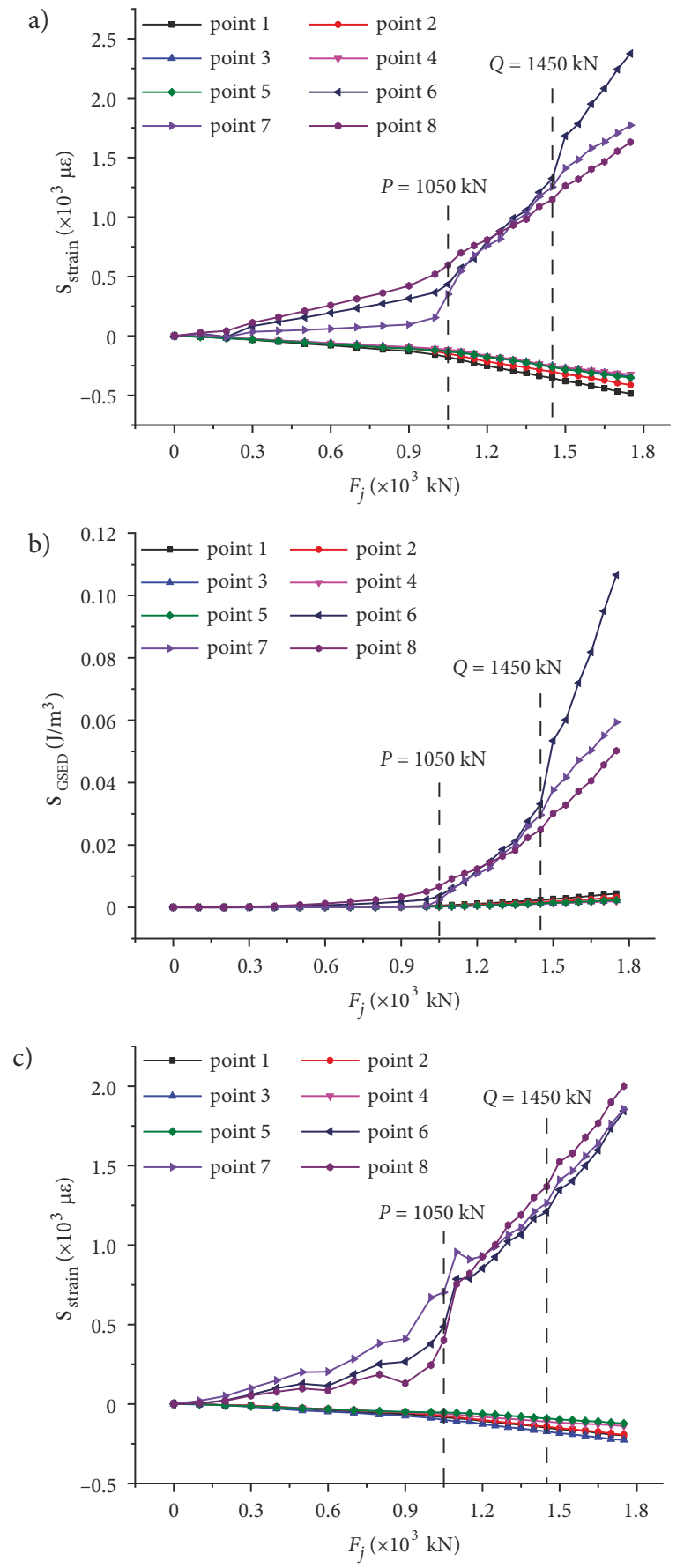

Figure 7. To be continued 
d)

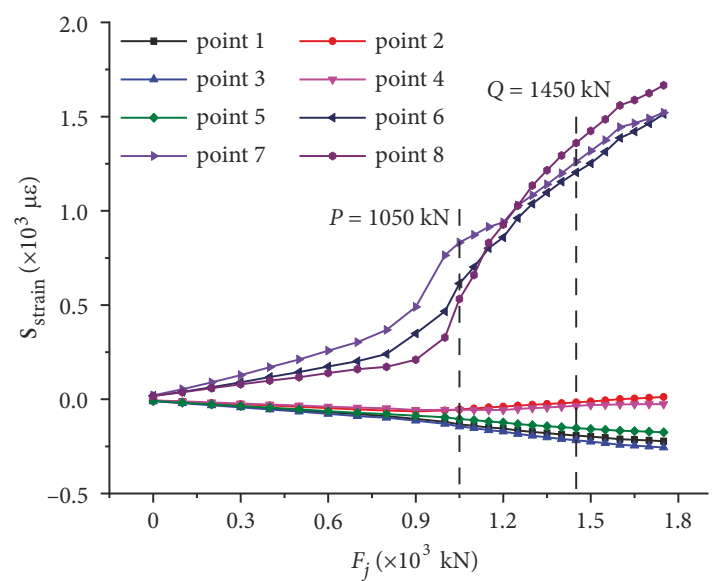

Figure 7. The curves of strain-based and GSED-based stressing state modes with respect to load for cross section $B$ : (a) the $\mathbf{S}_{\text {strain }}-F$ curve for surface concrete; (b) the $\mathbf{S}_{\mathrm{GSED}}-F$ curve for surface concrete; (c) the $\mathbf{S}_{\text {strain }}-F$ curve for steel bars; (d) the $\mathbf{S}_{\text {strain }}-F$ curve for internal concrete

2. But, the stressing state modes in Figure $7(\mathrm{c})$ and (d) do not embody the leap feature at characteristic loads $P$ and $Q$, and their mutations occur before $P$ and $Q$, suggesting that the failure of the bridge model is characterized by the structural outside concrete in the sense of structural stressing state;

a)

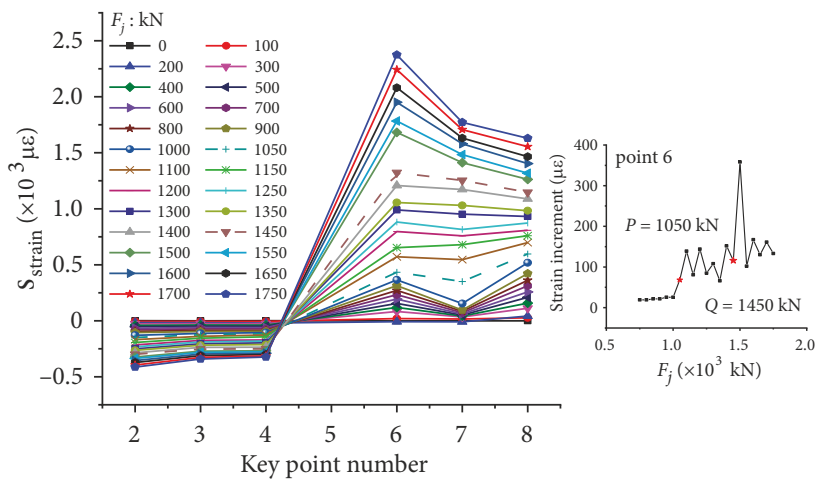

3. Besides, the strains and GSEDs in the soleplate are greater than those in the roof during the loading process, implying that the responses of concrete in two locations (surface and soleplate) play control roles for the updated failure load.

Also, the strain-based or GSED-based stressing state modes $\left(\mathbf{S}_{\text {strain }}\right.$ or $\left.\mathbf{S}_{\mathrm{GSED}}\right)$ can be plotted in the other form. For instance, the change of the stressing state modes in Figure 7(a) and (c) can be demonstrated as the forms in Figure 8 . The dash curves indicate that the leap features of stressing state modes are consistent with those revealed in Figure 6. Correspondingly, the strain increment of the critical point in the stressing state mode also reflects the leap features at characteristic loads $P$ and $Q$, and can be used as a parameter characterizing the stressing state mode $\mathbf{S}_{\text {strain }}$.

\subsection{Stressing state mode for sectional location}

In order to further reveal the mutation characteristics of the bridge model, this study builds the strain-based stressing state modes for the web location and the mid location on the key cross section B and plots the curves of the modes to load increase, as shown in Figure 9. Each stressing state mode includes four strain measuring points, two at the top and bottom concrete surfaces and two on the

b)

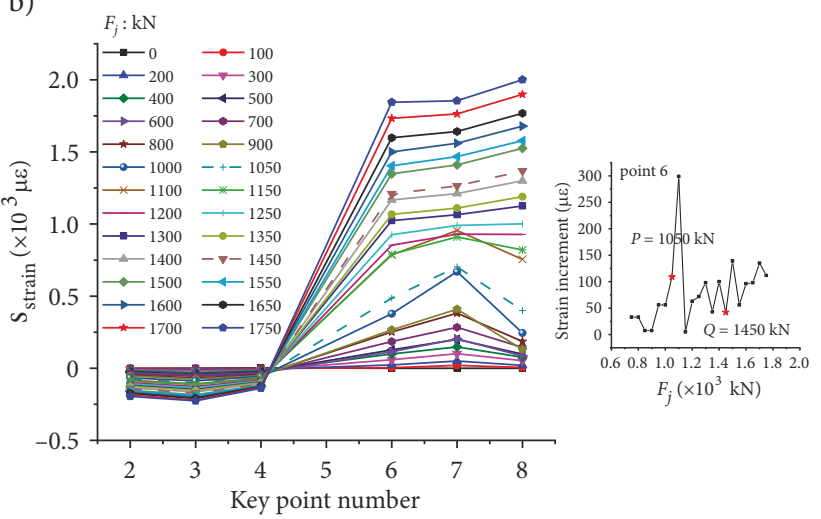

Figure 8. The changing feature of strain-based stressing state modes for cross sections: (a) the change of stressing state mode for the surface concrete of section B; (b) the change of stressing state mode for the steel bars of section B.
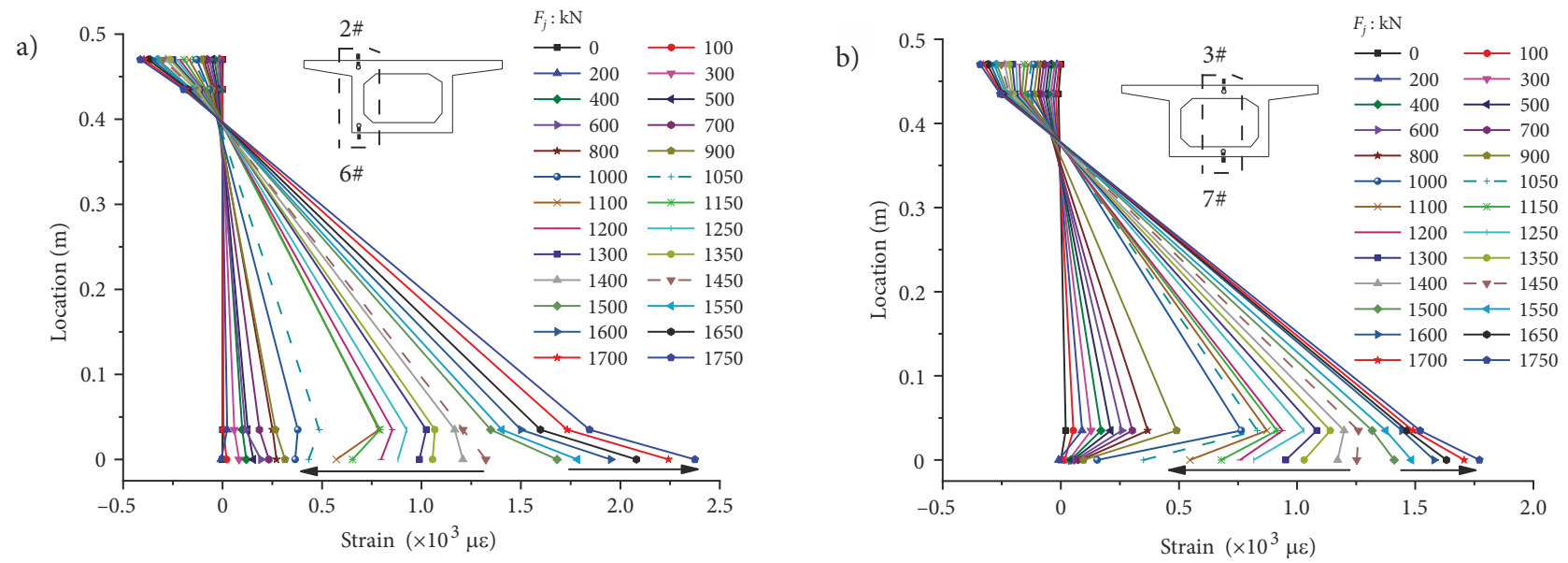

Figure 9. Location-strain curve of cross section B: (a) the changing feature of the web location's stressing state; (b) the changing feature of the mid location's stressing state 
steel bars in the roof plate and the soleplate, respectively. Figure 9(a) shows that the strains at the soleplate grow obviously faster than those at the roof. Before $800 \mathrm{kN}$, the strains at 4 points are basically in a straight line, which shows that this zone in section B maintains flat state during this load-bearing stage. After $800 \mathrm{kN}$, the strain growth at point 6 on the soleplate surface is slightly slower than that in the internal steel bar, indicating that the flat state of the section begins to embody a slight nonlinear state but still keeps the basic flat state. This feature could be that the bridge model produces some elastic-plastic deformation; Figure 9(b) shows that the flat state change of the section emerges at $900 \mathrm{kN}$, indicating that the elastic-plastic deformation will gradually develop to other locations. When a certain number of locations enter elastic-plastic working state, the bridge model's stressing state will mutate from the linear-elastic working state to the elastic-plastic working state at characteristic load $P$. In other words, when the load is exerted to characteristic load $P$, the qualitative change of the sectional flat state takes place, implying that this section has not been the basic flat state instead of a non-flat state. Hence, it could be reasonable to define load $P$ as the elastic-plastic branching point, signifying that the stressing state mode of the whole structure begins to change from the elastic state into elastic-plastic state.

\section{Analysis of stressing state characteristic parameters}

\subsection{Characteristic parameters for different materials and key points}

In the experiment, strain gauges are placed on the steel bars at the eight key points inside sections $\mathrm{B}$ and $\mathrm{D}$, together with the embedded concrete strain gauges there. Thus, an analysis can be proceeded to see the bonding performance between steel bar and concrete from the experimental strain data. Figure 10 gives out several Strain- $F$ curves of steel and concrete at points 7, 8 and 9. From Figure $10(a)-(c)$, the bonding performance between steel bar and concrete can be divided into three stages with different features. Before characteristic load $P$ (structur-

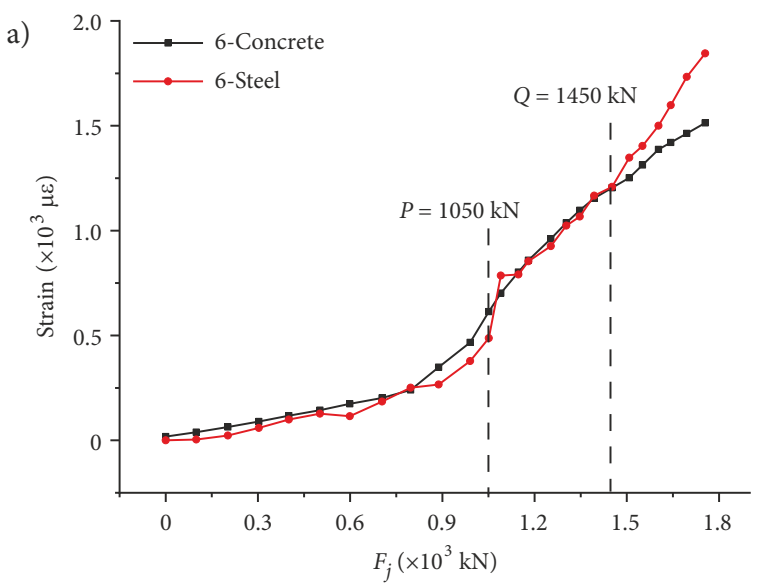

Figure 10. To be continued
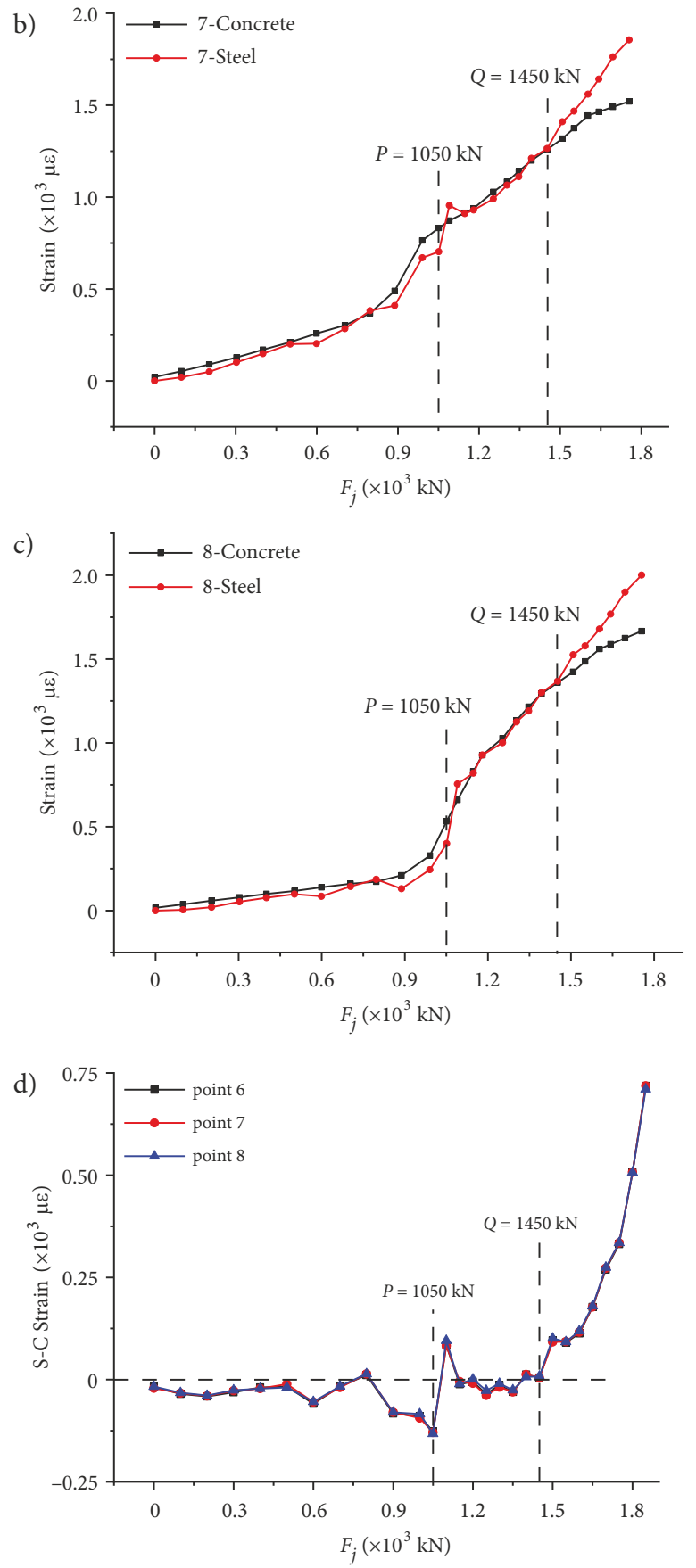

Figure 10. The bonding performance between steel bar and concrete: (a) Strain- $F$ curves of steel and concrete at points 7 curve; (b) Strain- $F$ curves of steel and concrete at points 8; (c) Strain- $F$ curves of steel and concrete at points 9; (d) S-C Strain (the difference value of stain between steel and concrete)- $F$ curves at points 7,8 and 9

al elastic stressing state), the bonding between steel bar and concrete is adjusted until a quite tight state or a consistent trend. Then, from load $P$ on state), the bonding keeps an ideal state until characteristic load $Q$ (starting point of structural failure). Finally, from failure load $Q$, the bonding starts to be broken off gradually, embodying the bifurcate feature. Evidently, the characteristic loads $P$ and $Q$ also characterize and define three bonding states between steel and concrete. In addition, from the curves 
of the difference (S-C Strain) between the strains of steel and concrete to load also reflect the qualitative mutation characteristics of the bonding performance at characteristic loads $P$ and $Q$, as shown in Figure 10(d).

\subsection{Characteristic parameters for bending and axial stressing states}

Under the given vertical loads, the bridge model withstands in-plane and out-plane bending moment, torsional moment and axial force. In this regard, the corresponding characteristic parameters of different internal forces are proposed to see their changing features around characteristic loads $P$ and $Q$. Due to the limited strain data, it can just structure the generalized characteristic parameter for in-plane, out-plane bending and axial force. Eqn (9) is proposed to calculate the difference between the strains at the vertical points in the soleplate and roof, in order to express the in-plane bending behavior:

$$
\Delta \varepsilon_{i}=\varepsilon_{i}^{s}-\varepsilon_{i}^{r},
$$

where $i$ is $1,2,3$, denotes concrete surface, steel bars and internal concrete, respectively; $\varepsilon_{i}^{s}$ is the strain at the ith location on the soleplate; $\varepsilon_{i}^{r}$ is the strain at the $i$ th location on the roof. Figure 11 illustrates that the in-plane and outplane bending changes as well as the axial force change also embody the bifurcate and mutation features around characteristic loads $P$ and $Q$. In addition, the bonding performance between steel bar and concrete is also embodied consistent with that mentioned above.

\subsection{Characteristic parameters of coordinative working state}

Here an investigation is tried to see whether structural coordinate working behavior could be reflected in a sense of structural stressing state or not. From this consideration, the ratio between the GSED sum of each sub part and the total of GSEDs for all the sub parts is proposed as Eqn (9):

$$
\rho_{i}=\frac{E_{i}}{E},
$$

where $\rho_{i}$ is the coordinate working parameter of the $i$ th sub parts; $E_{i}$ is the sum of GSED values in the $i$ th sub part; $E$ is the sum of GSED values of all sub parts. Under the load-bearing process, any two sections in the bridge model interact on each other. So two key cross sections B and $\mathrm{D}$ are chosen to make their coordinate parameters to see the bridge model's coordinate working features. The bridge model mainly suffers the bending moment under the given loading case. Figure 12 shows the change of coordinate working parameters (GSED ratios) with load increase, indicating that there are three stressing state stages basically consistent with the bridge model's stressing state feature revealed above. The coordinate working parameters of sections $\mathrm{B}$ and $\mathrm{D}$ embody obvious differences in the three stages. In the elastic stage before load $P$, the GSED a)

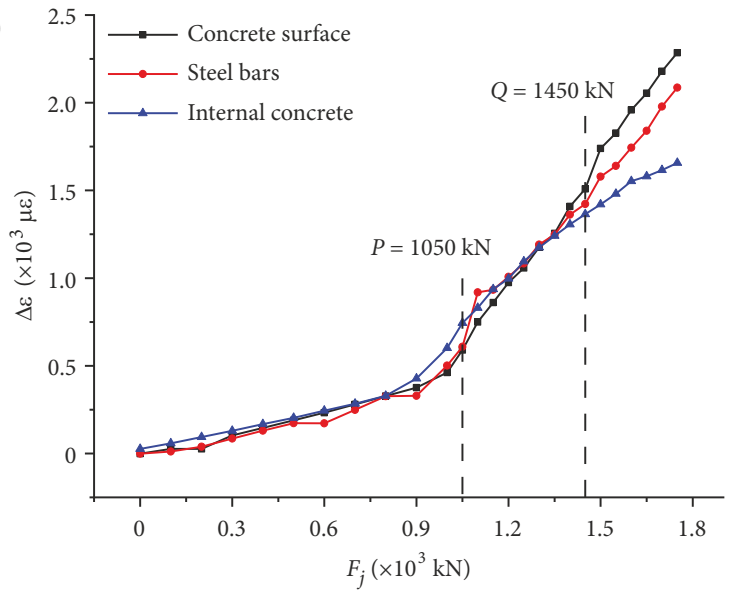

b)

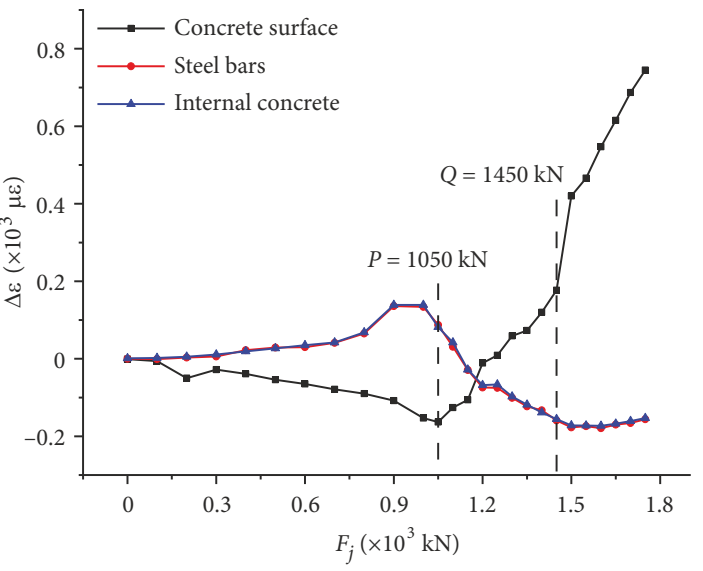

c)

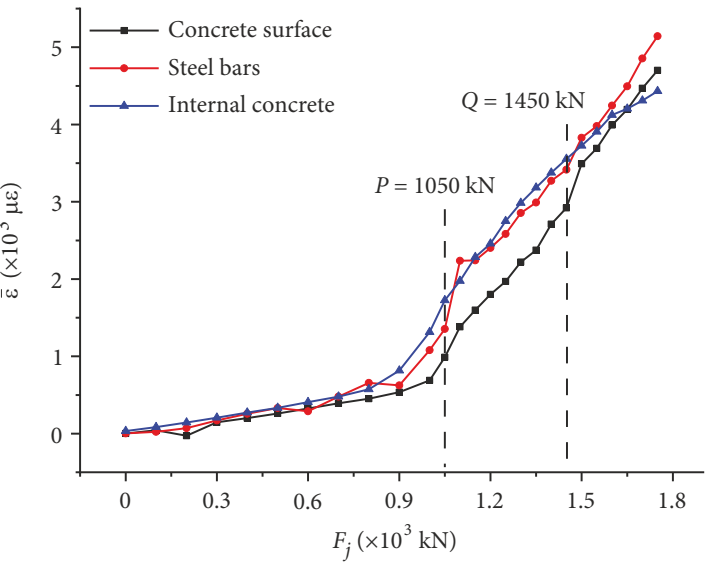

Figure 11. The changing features of generalized internal forces: (a) generalized in-plane bending; (b) generalized out-plane bending; (c) generalized axial force

ratios of the mid-fulcrum section $\mathrm{D}$ keep stable, but higher than that of section B, even over $60 \%$. Besides, it still implies that the slight plastic deformation close to load $P$ could be a revelation of structural self-adjusting ability to enhance structural coordinate working performance. But in the elastic-plastic stage between characteristic loads $P$ and $Q$, the GSED ratios of the midspan section $B$ is quite close to that of section $\mathrm{D}$, less than $10 \%$. In the bridge model's failure stage from load $Q$ on, the GSED ratios quickly develop to the opposite directions. 


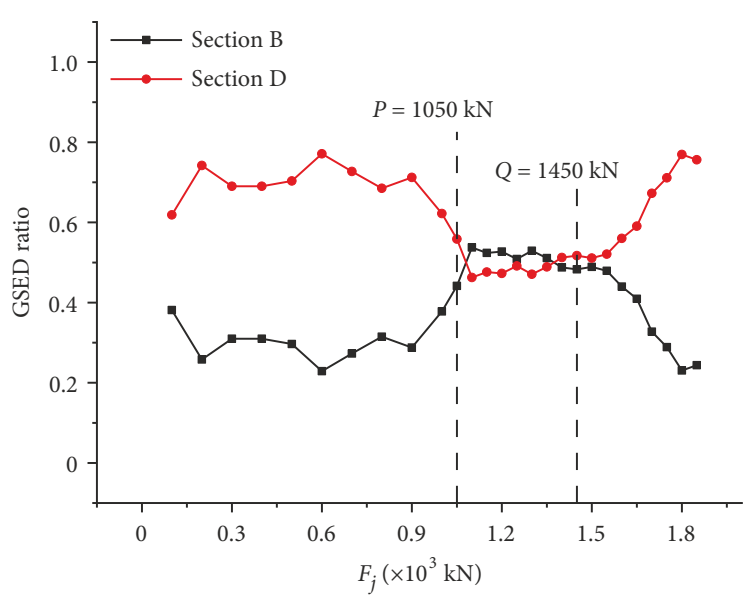

Figure 12. The changing feature of GSED ratios for sections $B$ and $D$

\section{Conclusions}

The investigation into the working behavior of a large curvature continuous prestressed concrete box-girder bridge model reveals its stressing state's qualitative leap feature at a certain load $Q$. This essential leap feature implies the starting point of the bridge model's failure process. Hence, the definition of the bridge model's failure load is updated at load $Q$, which could further lead to the update of the relative design codes.

Characteristic loads $P$ and $Q$ detected by the M-K criterion qualitatively divide three stressing state stages in the load-bearing process of the bridge model. Correspondingly, the stressing state modes composed of strains and GSEDs for structural components, as well as the stressing state modes for different types of internal forces, evidently embody the leap features around loads $P$ and $Q$. Furthermore, the bonding feature between steel bar and concrete can be revealed through the proposed characteristic parameter, which is consistent with three characteristic stages of the bridge model's stressing state. Finally, the coordinate working feature of two key sections in the bridge model can be reflected through the proposed GSED ratios.

In a sense, the analysis of the bridge model's stressing state explores a new way to structural analysis and provides the rational reference to the improvement of structural design.

\section{Acknowledgements}

This work was financially supported by the National Natural Science Foundation of China (Grant No. 51608069), the Chongqing Basic and Frontier Research Projects of China (Grant No. cstc2015jcyjys0011) and the Fundamental Research Funds for the Central Universities (Grant No. 106112016CDJXY200002). The authors would like to express their gratitude to Peng Dai for carrying out the excellent bridge model experiment and giving the experimental data in detail in his dissertation thesis. The authors would also like to thank the members of the HIT 504 office for their selfless help and useful suggestions.

\section{Funding}

This work was supported by the $<$ National Natural Science Foundation of China> under Grant [number 51608069]; $<$ Chongqing Basic and Frontier Research Projects of China $>$ under Grant [number cstc2015jcyjys0011]; < Fundamental Research Funds for the Central Universities> under Grant [number 106112016CDJXY200002].

\section{Author contributions}

Zhou Guangchun and Shi Jun conceived the study and were responsible for the design and development of the data analysis. Qin Fengjiang and Shi Jun were responsible for data collection, analysis and interpretation. Shen Jiyang and Li pengcheng helped perform the analysis with constructive discussions. Shen Jiyang wrote the first draft of the article.

\section{Disclosure statement}

All the authors have no conflict of interest.

\section{References}

Bu, L. T., \& Zheng, M. H. (2017). Study on construction monitoring of curved prestressed concrete roof box girder. Hunan Daxue Xuebao/Journal of Hunan University Natural Sciences, 44(7), 78-85.

https://doi.org/10.16339/j.cnki.hdxbzkb.2017.07.010

Cai, Z. B. (2013). The study on torsional effect of highway concrete curved box-girder bridge ( $\mathrm{PhD}$ Thesis, Central South University).

Cheung, M. S., \& Cheung, Y. K. (1984). Analysis of curved box girder bridges by finite strip method. International Association of Bridge and Structural Engineers (IABSE) Publications, 31(1), 1-8.

Dai, P. (2007). Experimental study and numerical analysis on bearing reaction and bearing capacity of curved prestressed concrete box girder bridge (PhD Thesis, Chang'an University) (in Chinese).

Dabrowski, R. (1968). Curved thin-walled girder, theory and analysis. New York: Springer-Verlag New York, Inc.

Hirsch, R. M., Slack, J. R., \& Smith, R. A. (1982). Techniques of trend analysis for monthly water quality data. Water Resources Research, 18(1), 107-121.

https://doi.org/10.1029/wr018i001p00107

Huang H. X., Liu, B., Zhang, Y., \& Tian, X. Y. (2009). Bearing reaction test and analysis of prestressed concrete curved girder bridge. In Proceeding of $8^{\text {th }}$ International Symposium on Test and Measurement (pp. 732-734). Chongqing, China.

Huang, Y., Yu, Z., \& Liu, C. (2014). Method for predicting failure load of masonry wall panel based on generalized strain energy density. Journal of Harbin Institute of Technology, 140(8), 04014061.

https://doi.org/10.1061/(asce)em.1943-7889.0000771

Jiang, X. D. (2008). Design analysis of prestressed concrete curve box-girder bridge and its application (MA Thesis, Zhejiang University) (in Chinese).

Kendall, M. G. (1990). Rank correlation methods. New York: Oxford University Press. https://doi.org/10.2307/2333282

Kim, K., \& Yoo, C. H. (2006). Effects of external bracing on horizontally curved box girder bridges during construction. Engineering Structures, 28(12), 1650-1657.

https://doi.org/10.1016/j.engstruct.2006.03.001 
Khaloo, A. R., \& Kafimosavi, M. (2007). Enhancement of flexural design of horizontally curved prestressed bridges. Journal of Bridge Engineering, 12(5), 585-590. https://doi.org/10.1061/(asce)1084-0702(2007)12:5(585)

Khan, E., Lobo, J. A., \& Linzell, D. G. (2018). Live load distribution and dynamic amplification on a curved prestressed concrete transit rail bridge. Journal of Bridge Engineering, 23(6), 04018029 .

https://doi.org/10.1061/(asce)be.1943-5592.0001236

Lin, T. Y., \& Burns, N. (1983). Prestressed concrete structure design. China Railway Publishing House (in Chinese).

Mann, H. B. (1945). Nonparametric tests against trend. Econometrica, 3(3), 245-259. https://doi.org/10.2307/1907187

Meyer, C., \& Scordelis, A. C. (1970). Computer program for prismatic folder plates with plate and beam elements. (Structural Engineering and Structural Mechanics Report No. SESM 703). University of California, Berkeley.

Przemysław, M., Wojciech, T., \& Radomski, W. (2017). The impact of using concrete of various density on the state of stresses in prestressed concrete flyovers over highways. Procedia Engineering, 193, 258-265. https://doi.org/10.1016/j.proeng.2017.06.212

Qiao, J. L., Jin, Y., Tian, W. L., \& Li, F. (2012). The analysing of coupled bending torsion and shear-lag of curved box girder bridge considering prestress and initial curvature. Advanced Materials Research, 446-449, 3360-3364.

https://doi.org/10.4028/scientific5/amr.446-449.3360

Rogers, L. P., \& Seo, J. (2016). Vulnerability sensitivity of curved precast-concrete I-girder bridges with various configurations subjected to multiple ground motions. Journal of Bridge Engineering, 04016118.

https://doi.org/10.1061/(asce)be.1943-5592.0000973

Seo, J.; \& Rogers, L. P. (2017). Comparison of curved prestressed concrete bridge population response between area and spine modeling approaches toward efficient seismic vulnerability analysis. Engineering Structures, 150, 176-189.

https://doi.org/10.1016/j.engstruct.2017.07.033

Seo, J., Rogers, L. P., \& Hu, J. W. (2018). Computational seismic evaluation of a curved prestressed concrete I-girder bridge equipped with shape memory alloy. European Journal of Environmental and Civil Engineering. https://doi.org/10.1080/19648189.2018.1492972
Shi, S. W., \& Xiang, Z. F. (2012). Bridge structure test and detection technology. Chongqing University Press (in Chinese).

Shi, X., Cao, Z., Ma, H., \& Ruan, X. (2018). Failure analysis on a curved girder bridge collapse under eccentric heavy vehicles using explicit finite element method: case study. Journal of Bridge Engineering, 23(3), 05018001. https://doi.org/10.1061/(asce)be.1943-5592.0001201

Timoshenko, S. P., \& Gere, J. M. (1961). Theory of elastic stability ( $2^{\text {nd }}$ ed.). New York: McGraw HilBook Co. Inc.

Vlasov, V. Z. (1961). Thin-walled elastic beam. Washington: National Science Foundation.

Walter, P. (1985). The cause of cracking in post-tensioned concrete box girder bridges and retrofit procedures. PCI Journal, 30(2), 82-139. https://doi.org/10.15554/pcij.03011985.82.139

Yang, Z. Y., Zhao, Y., \& Liu, Z. S. (2011). Research on stretching order of tendons in PC curved box girder bridge. Advanced Materials Research, 219-220, 487-491. https://doi.org/10.4028/www.scientific.net/amr.219-220.487

Yuan, A., Dai, H., \& Sun, D. (2013). Behaviors of segmental concrete box beams with internal tendons and external tendons under bending. Engineering Structures, 48, 623-634. https://doi.org/10.1016/j.engstruct.2012.09.005

Zhang, G. Y. (2002). Bridge structure test. People's Traffic Press (in Chinese).

Zhang, Y., Zhou, G. C., Xiong, Y., \& Rafiq, Y. (2010). Techniques for predicting cracking pattern of masonry wallet using artificial neural networks and cellular automata. Journal of Computing in Civil Engineering, 24(2), 161-172. https://doi.org/10.1061/(asce)cp.1943-5487.0000021

Zhou, G. C., Rafiq, M. Y., Bugmann, G., \& Easterbrook, D. J. (2006). Cellular automata model for predicting the failure pattern of laterally loaded masonry wall panels. Journal of Computing in Civil Engineering, 20(6), 400-409. https://doi.org/10.1061/(asce)0887-3801(2006)20:6(400)

Zhou, G. C., Pan, D., Xu, X., \& Rafiq, Y. M. (2010). An innovative technique for predicting failure/cracking load of masonry wall panel under lateral load. Journal of Computing in Civil Engineering, 24(4), 377-387.

https://doi.org/10.1061/(asce)cp.1943-5487.0000040 